\title{
Physicochemical and morphological transformations of chitosan nanoparticles across the gastrointestinal tract and cellular toxicity in an in vitro model of the small intestinal epithelium
}

Zhongyuan Guo ${ }^{1}$, Xiaoqiong $\mathrm{Cao}^{1}$, Glen M DeLoid ${ }^{1}$, Kaarunya Sampathkumar ${ }^{2}, \mathrm{Kee}$ Woei $\mathrm{Ng}^{2}$, Say Chye Joachim Loo $^{2}$, and Demokritou Philip ${ }^{1 *}$

${ }^{1}$ Center for Nanotechnology and Nanotoxicology, Department of Environmental Health, Harvard T. H. Chan School of Public Health, Boston, MA 02115, USA

${ }^{2}$ School of Materials Science and Engineering, Nanyang Technological University 50 Nanyang Avenue 639798, Singapore, Singapore

*Corresponding author: Philip Demokritou, PhD, Phone: +1 617432 3481; Email: pdemokri@hsph.harvard.edu 


\begin{tabular}{ccccccc}
\hline Condition & Material & $\begin{array}{c}\mathbf{D}_{\mathbf{3}, \mathbf{2}} \\
(\boldsymbol{\mu m})\end{array}$ & $\begin{array}{c}\mathbf{D}_{\mathbf{4}, \mathbf{3}} \\
(\boldsymbol{\mu m})\end{array}$ & $\begin{array}{c}\mathbf{D v}_{\mathbf{1 0}} \\
(\boldsymbol{\mu m})\end{array}$ & $\begin{array}{c}\mathbf{D v}_{\mathbf{5 0}} \\
(\boldsymbol{\mu m})\end{array}$ & $\begin{array}{c}\mathbf{D v}_{\mathbf{9 0}} \\
(\boldsymbol{\mu m})\end{array}$ \\
\hline pH7 & Chp & $2.79 \pm 0.01$ & $25.5 \pm 0.4$ & $1.35 \pm 0.01$ & $3.24 \pm 0.01$ & $91.3 \pm 3.4$ \\
Water & Chnp & $2.31 \pm 0.01$ & $3.34 \pm 0.01$ & $1.28 \pm 0.01$ & $2.69 \pm 0.01$ & $5.94 \pm 0.01$ \\
& SS-Chnp & $5.01 \pm 0.03$ & $222 \pm 5$ & $1.80 \pm 0.01$ & $10.8 \pm 0.4$ & $731 \pm 14$ \\
pH7 & Chp & $2.79 \pm 0.01$ & $41.4 \pm 0.1$ & $1.30 \pm 0.01$ & $3.24 \pm 0.01$ & $183 \pm 1$ \\
Oral & Chnp & $2.35 \pm 0.00$ & $3.50 \pm 0.00$ & $1.28 \pm 0.01$ & $2.73 \pm 0.00$ & $6.32 \pm 0.01$ \\
& SS-Chnp & $4.86 \pm 0.11$ & $168 \pm 30$ & $1.80 \pm 0.01$ & $9.52 \pm 1.59$ & $557 \pm 95$ \\
pH1.4 & Chp & $0.0818 \pm 0.0043$ & $0.289 \pm 0.026$ & $0.0312 \pm 0.0013$ & $0.165 \pm 0.015$ & $0.740 \pm 0.070$ \\
Gastric & Chnp & $2.96 \pm 0.01$ & $15.7 \pm 0.3$ & $1.30 \pm 0.01$ & $4.02 \pm 0.01$ & $27.7 \pm 0.3$ \\
pH7 & SS-Chnp & $5.08 \pm 0.02$ & $115 \pm 4$ & $1.94 \pm 0.01$ & $8.05 \pm 0.11$ & $394 \pm 12$ \\
Small & Chp & $8.46 \pm 0.11$ & $466 \pm 14$ & $2.53 \pm 0.02$ & $163 \pm 10$ & $1396 \pm 43$ \\
Intestine & SS-Chnp & $3.48 \pm 0.04$ & $207 \pm 19$ & $1.44 \pm 0.01$ & $4.59 \pm 0.11$ & $819 \pm 129$ \\
\hline & & $4.83 \pm 0.06$ & $219 \pm 17$ & $1.76 \pm 0.01$ & $9.70 \pm 0.71$ & $718 \pm 50$ \\
\hline
\end{tabular}

Table S1. Colloidal properties of chitosan materials dispersed in water $\mathbf{p H}$ buffers. $\mathrm{Dv}_{50}$ : the size at which $50 \%$ of the sample is smaller and $50 \%$ is larger, or the median of the volume distribution; $\mathrm{Dv}_{10}$ : the size below which $10 \%$ of the total sample volume lies; $\mathrm{Dv}_{90}$ : the size below which $90 \%$ of the sample volume distribution lies; $\mathrm{D}_{4,3}$ :volume-weighted mean; $\mathrm{D}_{3,2}$ : surface-weighted mean. Results are presented as mean $\pm \mathrm{SEM}, \mathrm{n}=5$ independent measurements. 

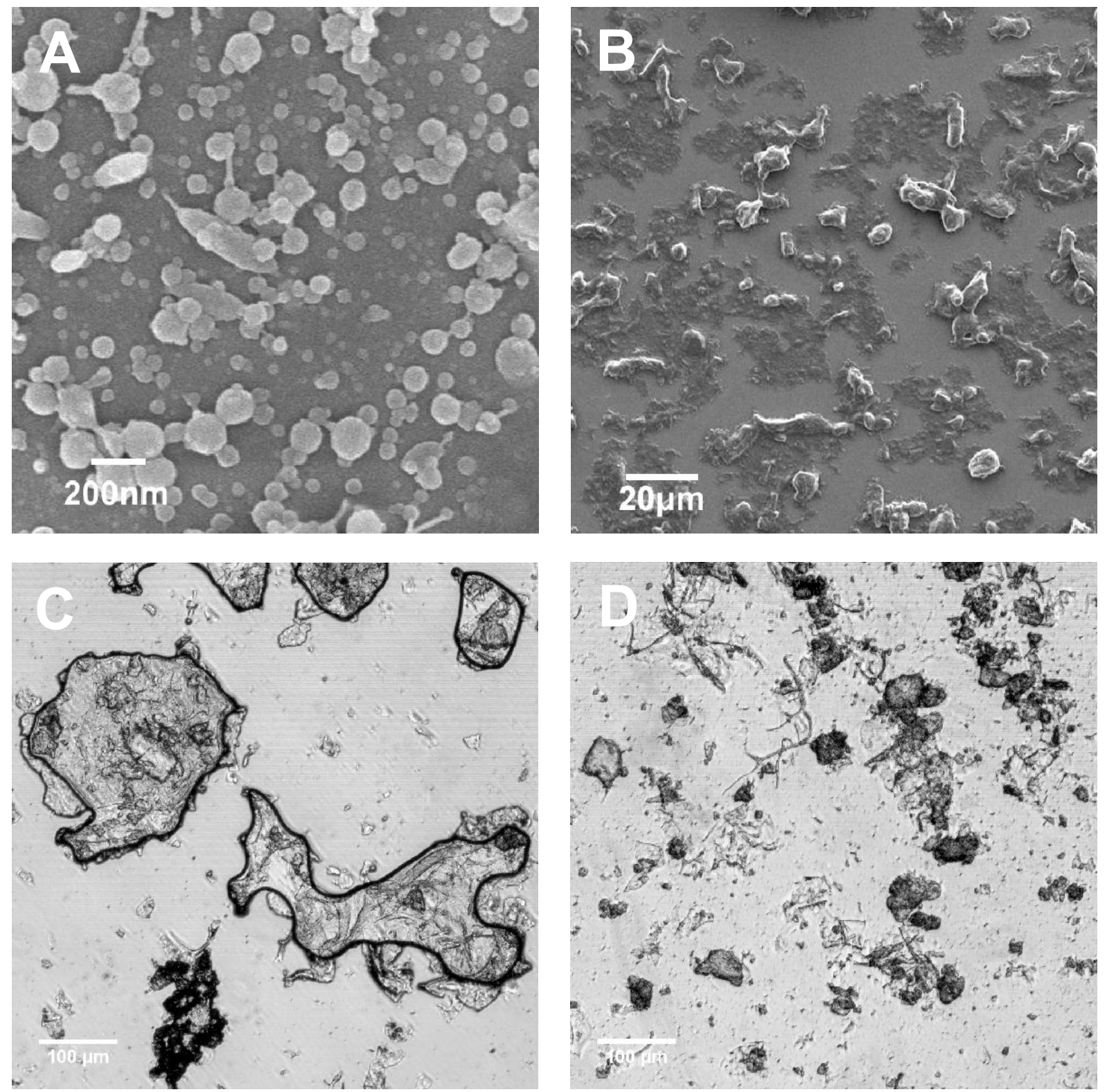

Figure S1 Morphology of chitosan nanoparticles (Chnp) and starch coated Chnp (SS-Chnp) by scanning electron microscopy (SEM) and optical microscopy: A. SEM image of Chnp. B. SEM image of SS-Chnp. C. Optical image of unsonicated SS-Chnp. D. optical image of SSChnp after 1 min sonication. 